months over a 4-yr period. All graft failures are included. Survival in group $O$ and non-group $O$ recipients shows the same difference as in the Cambridge data, that is that grafts survive better in group $O$ patients. At 1 yr a $2 \times 2$ test shows formal significance, $\chi^{2}=6.49, \quad P=0.02-0.01$; however this test was only made after looking at the graphical output. The group A patients were divided according to whether they received group $\mathrm{O}$ or group $\mathbf{A}$ donor kidneys, Fig. $1 b$. There seems to be no initial difference in survival, but the numbers are small.

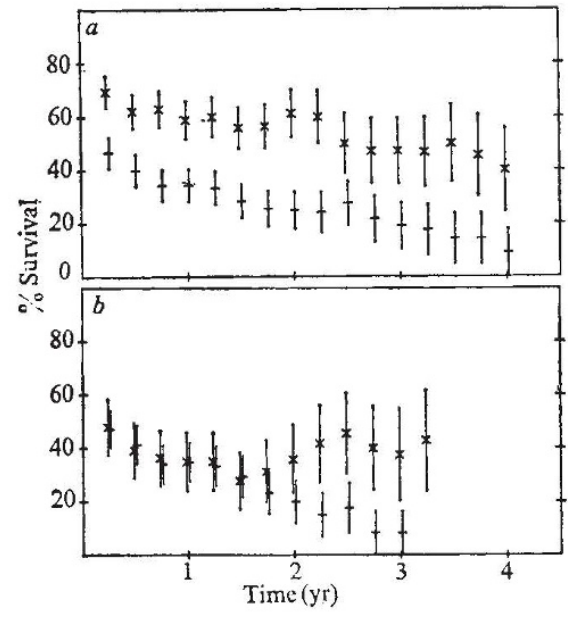

Fig. 1 Survival of kidney grafts in Birmingham. $a$, Survival in group $\mathrm{O}$ $(x)$ and group $\mathrm{A}(+)$ recipients. $b$, Subdivision of group A recipients according to whether they received group $\mathrm{O}(x)$ or group $\mathrm{A}(+)$ kidneys. There were 62 group $O$ recipients; the 75 group $A$ recipients received 51 group $A$ and 23 group $O$ kidneys, and one of unknown blood group. Vertical bars represent standard error.

Only group $O$ recipients are guaranteed an identical ABO match. If a mismatch for the product of the $O$ gene is not acceptable, using the genotype frequencies for southern England from Race and Sanger ${ }^{2}$, this will only account for $14.4 \%$ of A to A transplants and $17.4 \%$ of $\mathrm{O}$ to A transplants because the majority of group As will be heterozygous AO Is this, together with an allowance for $A_{1}$ and $\mathrm{A}_{2}$ mismatching, enough to account for the observed discrepancy in graft survival between group $\mathrm{O}$ and group $\mathrm{A}$ recipients? If $A B O$ genotype is important, then without $\mathbf{A}_{1}$ and $\mathbf{A}_{2}$ grouping and matching, only $44 \%$ of $A$ to $A$ grafts will be genotypically identical.

I am indebted to Professor J. H. Edwards and Mrs Karen Glenn for developing the system of computer documentation.

I thank Mr A. D. Barnes for making the survival information available and the Kidney Research Fund for financial assistance with the analysis. Yours faithfully, Pauline Mackintosh

Regional Transfusion Service,

Vincent Drive,

Birmingham B15 2SG, UK

1 Joysey, V. C., Roger, J. H., Evans, D. B., and Herbertson, B. M., Nature, 246, 163 (1973).

${ }^{2}$ Race, R. R., and Sanger, R., Blood Groups in Man (Blackwell Scientific, Oxford and Edinburgh, 1968).

\section{Spittlebug morph mimics avian excrement}

Sir,-Most colour forms ${ }^{1.2}$ of the spittlebug, Philaenus spumarius, are cryptic, but the marginella morph has a black body outlined by white. Thompson ${ }^{3}$ has proposed that this phenotype represents an example of escape warning coloration, suggesting that experienced predators ignore marginella individuals because they remember previous "frustrating encounters" with marginella more vividly than similar encounters with cryptic morphs. The logic of this argument is tenuous. It seems equally likely that predators should chase marginella more actively because of an equally vivid recollection of successful encounters. Moreover, if the phenotype was a warning pattern its fitness should increase with increased frequency, for a greater proportion of experienced predators would be encountered. Both cryptic and marginella forms must be present for such conditioning, but this is assured even when the marginella allele is fixed, for only the females are brightly coloured, males are cryptic $^{2}$. On this basis fixation of the marginella allele should occur, yet in natural populations its frequency remains low.

Patterns of black and white have functions other than predator warning; among small arthropods such patterns are frequently associated with excrement mimicry ${ }^{4}$. Indeed the marginella pattern, coupled with the elongate shape of $P$. spumarius, produces a convincing resemblance to a bird dropping. lgnoring possible complications, such as the occurrence of other excrement mimics or changes in the grouped abundance of the cryptic morphs, marginella frequency should be intimately related to model abundance. A uniform distribution of bird droppings could, for instance, be the cause of the relatively constant frequency of the marginella morph in the Great Lakes area of North America?.

The frequency-dependent fitness of mimetic forms can lead to sex limitation when the timing of predation differs between sexes. For example, if predation is postreproductive in males, but prereproductive in females, suppression of male mimicry allows population size to increase while not affecting male fitness. This situation is closely approximated in $P$. spumarius as mating occurs in the early summer while egg deposition is delayed until autumn ${ }^{5}$. It is not surprising then, that modifiers have accumulated to restrict the marginella pattern to females.

In conclusion there seems little reason to suspect that the marginella phenotype has any warning significance; rather it mimics avian excrement in a situation analogous to classical Batesian mimicry. Yours faithfully,

Paul Hebert

School of Biological Sciences, University of Sydney, Sydney, NSW 2006, Australia

${ }^{1}$ Weaver, C. R., and King, D. R., Ohio Agr. Exp. Sta. Res. Bull. 741, 1 (1944).

${ }^{2}$ Thompson, V., and Halkka, O., Am. Midl. Nat., 89, 348 (1973).

${ }^{3}$ Thompson, V., Nature, 242, 126 (1973).

${ }^{4}$ Cott, H. B., Adaptive Colouration in Animals (Methuen, London, 1940).

${ }^{5}$ Wiegert, R. G., Ecol. Monogr., 34, 217 (1964).

Mr THOMPSON REPLIES: I believe these objections to my proposal are not well founded. Cryptic coloration of all forms is not necessary for the maintenance of an apostatic polymorphism. This can be demonstrated quantitatively by a simple extension of the model of Clarke and $\mathrm{O}^{\prime}$ Donald ${ }^{1}$ for frequency-dependent selection at one locus with dominance. Let $A$ represent a dominant allele for conspicuous coloration with frequency $1-q$ and $A^{1}$ a recessive allele for more cryptic coloration with frequency $q$. Let $t$ and $t_{1}$ represent factors relating the frequency of each phenotype to its selective value, and let $I$ be a standard fitness.

\begin{tabular}{|c|c|c|}
\hline Genotypes & $A A$ and $A A^{1}$ & $A^{1} A^{1}$ \\
\hline $\begin{array}{l}\text { Frequency after } \\
\text { random mating }\end{array}$ & $1-q^{2}$ & $q^{2}$ \\
\hline \multicolumn{3}{|l|}{ Selective value: } \\
\hline $\begin{array}{ll}\text { Case 1 } & I-t \\
\text { Case 2 } & I-t\end{array}$ & $\begin{array}{l}\left.q^{2}\right) \\
\left.q^{2}\right)+w\left(1-q^{2}\right)\end{array}$ & $\begin{array}{l}I-t_{1} q^{2} \\
I-t_{1} q^{2}\end{array}$ \\
\hline
\end{tabular}

At equilibrium the selective values of the two phenotypes must be equal and for this example (Case 1) it can be shown that there is a non trivial stable equilibrium for the two alleles at $\hat{q}=\sqrt{[t]}$ $\left.\left(t+t_{1}\right)\right]$ provided that $t$ and $t_{1}$ are both positive (each colour form becomes less fit as it becomes more common). Both forms will be maintained in the population with the more conspicuous form at lower frequency.

Furthermore, an allele determining a conspicuous pattern which is also a warning pattern will not necessarily increase to fixation, even though the warning pattern per se confers greater fitness as its frequency increases. Let $w$ represent the relation between the frequency of the conspicuous phenotype and that part of its selective value determined by its function as a warning 